\title{
Atomic and electronic structures of a-SiC
}

\author{
V.I. Ivashchenko, V.I. Shevchenko \\ Institute of Problems of Materials Science, NAS of Ukraine, 3 Krzhyzhanovsky str., 03142 Kyiv, Ukraine \\ Phone: +380 (44) 411 3475; fax: +380 (44) 411 3475; e-mail: shev@celebris.materials.kiev.ua
}

\begin{abstract}
Molecular dynamics (MD) simulations based on an empirical potential approach have provided detailed information about chemical ordering and the structural short-range order in stoichiometric amorphous silicon carbide (a-SiC). Recursion band structure calculations based on amorphous geometries obtained from the MD simulations have enabled one to ascertain the mechanism of an influence of homopolar bonds, three-fold $\left(\mathrm{T}_{3}\right)$ and five-fold $\left(\mathrm{T}_{5}\right)$ coordinated defects, strongly disordered four-fold coordinated sites $\left(\mathrm{T}_{4}\right)$ and atoms, which are first nearest neighbors of these defects influencing on the distribution of electronic states. We have found that electronic states at the middle of the gap can be associated with these kinds of defects with the exception of antisite defects (like-atom or homopolar bonding). It is the problem of chemical ordering in the stoichiometric amorphous siliconcarbon alloy that is the main subject of the present work. In contrast to crystalline $\mathrm{SiC}$, in a-SiC, the resonance states at the valence band top associated to $\mathrm{Si}$-Si homonuclear bonds split for the low symmetry amorphous surrounding, which gives rise to the additional split states at the band gap bottom. As a result, in the amorphous material, the decrease of chemical ordering is accompanied by narrowing the band gap. The suggested band model of a-SiC agrees rather well with the available experimental results on the electronic distribution in this alloy.
\end{abstract}

Keywords: amorphous silicon carbide, chemical ordering, molecular dynamics, recursion method.

Paper received 21.01.02; revised manuscript received 26.02.02; accepted for publication 05.03.02.

\section{Introduction}

Understanding of properties of amorphous silicon carbide alloys $\left(\mathrm{a}-\mathrm{Si}_{1-\mathrm{x}} \mathrm{C}_{\mathrm{x}}\right)$ has progressed rapidly in recent years, motivated by the application of these alloys in wear-resistant coatings and electronic devices. One of the major problems consists in clarifying the interrelation among structural and electronic properties including the investigation of the effects of homonuclear bonding and other coordination defects on the electronic structure of tetrahedrally coordinated $\mathrm{sp}^{3}$-bonded a-Si ${ }_{1-\mathrm{x}} \mathrm{C}_{\mathrm{x}}$ alloys around the carbon concentration $\mathrm{x}=0.5$ at which the crystalline compound exists. A variety of structural studies have concluded that chemical ordering is incomplete and strongly dependent on deposition conditions. The experimental situation is nicely summarized in [1-3]. A degree of chemical ordering was found to range from near random to near complete. Coordination disorder is also found. $\mathrm{C}$ atoms have a tendency to form three-fold coordinated $\mathrm{sp}^{2}$ sites, especially in C-rich alloys. There are a few theoretical works devoted to examining the atomic distribution in $\mathrm{a}-\mathrm{Si}_{1-\mathrm{x}} \mathrm{C}_{\mathrm{x}}$ alloys, for $\mathrm{x}=0.5$ (a$\mathrm{SiC})[2,4,5]$. On the basis of ab initio non-local pseudopotential MD simulations, Finocchi et al. [2] have concluded that a-SiC does not exhibit chemical order and represents the tetrahedral network with the considerable part of $\mathrm{C}-\mathrm{C}$ homonuclear bonds (40$45 \%$ ). C atoms also give rise to both $\mathrm{sp}^{2}$ and $\mathrm{sp}^{3}$ sites, tend to form chainlike structures, surrounded by Si-C and $\mathrm{Si}$-Si network, where $\mathrm{Si}$ is distributed on distorted diamond-like sites. In contrast to this, Kelires [4] has found stronger chemical ordering, but with half the $\mathrm{C}$ atoms three-fold coordinated. The latter simulation has been performed in the framework of a continuousspace Monte Carlo method (MC) in combination with the potential of Tersoff [6]. Herewith, the carbon potential has been fitted to graphite. Tersoff [5] has used the same procedure, but the carbon empirical potential has been obtained from the experimental data for diamond. Tersoff's version has been fitted only to nonp-bonded structures, and gives an excellent description of $\mathrm{C}$ defects in $\mathrm{Si}[5]$. The recent total energy, calculation carried out within the pseudopotential-density-functional formalism (PDF) [7], has shown that partial chemical ordering is by far the most favored phase in a-SiC. Ordering in a-SiC has been studied in the framework of a free-energy model approach [8], 


\section{V.I. Ivashchenko et al.: Atomic and electronic structure of a-SiC}

based on the quasi-chemical approximation to the thermodynamics of regular solutions. It has been found that only $89 \%$ of all bonds are Si-C bonds, with about $5 \%$ of each of $\mathrm{Si}-\mathrm{Si}$ and $\mathrm{C}-\mathrm{C}$ bonds are present [8].

The density of states (DOS) of a-SiC has been investigated by Finocchi et al [2], Kelires and Denteneer $[7,9]$ in the framework of the PDF and Robertson [3] using the recursion method [10]. The latter work deals with modeling the DOS of a-SiC considering different substitution defects in 3C-SiC. The DOS obtained from pseudopotential calculations [2,7,9] does not exhibit a range of actually forbidden energies, which is due to the usual underestimation of the gap in the local density approximation. The effect of introducing homopolar bonds in a-SiC has been shown to be a drastic reduction of the energy gap and the disappearance of the ionicity gap at $\sim 11 \mathrm{eV}$.

In the present work, investigated were the effects of the chemical ordering and local geometry on the electronic structure, in particular, gap states (GS) of a-SiC alloy by means of MD simulations based on Tersoff's potential - recursion calculations. The obtained results were compared with the available experimental data.

\section{Computation details}

We have carried out MD simulations of computationally generating "samples" of a-SiC. Our samples have 64,216 or 512 atoms per cell, with periodic boundary conditions. The unit cell is simple cubic, with a volume, which corresponds to the density of crystalline $\mathrm{SiC}$ or is determined at constant pressure. The sample, representing zinc-blended (D) or rock-salt (B1) structure, was heated at constant volume or pressure to $8000 \mathrm{~K}$. The melt is then cooled at inverse rate of some $0.5 \times 10^{13} \mathrm{~K} / \mathrm{s}$ down to $300 \mathrm{~K}$. Both the melt and cooled sample were let free to evolve for $\sim 15 \mathrm{ps}$.

Since the MD simulations of a-SiC based on the atomic interactions which are modeled with the classical interatomic potential of Tersoff $[5,6]$ are performed for the first time, a definite correction of some potential parameters is needed. The application of the nonmodified potential in our MD simulation turned out to lead to the strong disordered structures with about $40 \%$ of silicon four-fold coordinated atoms. Consequently, this potential is unable to give appropriate results at the chosen conditions of the MD simulations. Keeping in mind that, in Tersoff's formalism, the cut-of parameters $\mathrm{R}$ and $\mathrm{S}$ were chosen somewhat arbitrarily and were not systematically optimized [5], we have carried out several calculations with various $\mathrm{R}$ and $\mathrm{S}$ parameters. The optimal amorphous structure with the maximum number of four-fold coordinated atoms turned out to be reached at $\mathrm{R} \sim 0.25 \mathrm{~nm}, \mathrm{~S} \sim 0.27 \mathrm{~nm}$. The results of the MD simulations of the 64-, 216- and 512-atom samples on the basis of the modified potential are summarized in Table 1. It should be noted that the corrects introduced into Tersoff's potential are obligate, since few-body classical potentials cannot describe correctly the large variety of different bonding situations encountered in MD simulations on going from the liquid to the amorphous and the crystal [11]. The samples with different degrees of chemical ordering have been obtained by means of varying the $\chi_{\mathrm{Si}-\mathrm{C}}$ parameter. The original, physical value $(=1.0086)$ of the $\chi_{\text {Si-C }}$ parameter is determined by fitting to the cohesive energy of $3 \mathrm{C}-\mathrm{SiC}[5]$.

The electronic structure of the alloy has been calculated using the tight-binding approximation using a basis set of the $\mathrm{sp}^{3} \mathrm{~s}^{*}$ scheme [12]. The parameters were basically those used in [3]. The $\mathrm{Si}-\mathrm{C}$ parameters have been modified to reproduce the valence bandwidth in 3C-SiC more accurately. The Si-C, Si-Si and C-C bond parameters have been superposed at the same scale according to Robertson's procedure. The density of states has been calculated directly using eigenvalues in 35 points of the irreducible segment of the Brilluion zone or using the recursion method (RM) of Haydock [10] and Nex [13 ]. The RM calculations have been carried out using the clusters of 4000-5000 atoms, retaining 54 levels of recursion coefficients.

\section{Results and discussion}

In Fig. 1 we present the pair correlation functions

Table 1. Characteristics of the samples with about 30\% of homopolar bonds obtained from 3C-SiC (D) and B1-SiC (B1) melts using the modified potential $\left(\chi_{\mathrm{Si}-\mathrm{C}}=1.0086\right)$. $\mathrm{N}_{\mathrm{a}}$ - the number of atoms in a sample; $\mathrm{a}_{0}$ - the side of the cubic cell; $\mathrm{R}_{\mathrm{Si}}$ - the cut-off distance of SiSi interactions; $n_{i}$ - the part of $i$-fold coordinated atoms; $N_{i}$ - the average coordination number of an atom $i$; $N_{i-j}$ - the part of $i-j$ bonds in the samples.

\begin{tabular}{lllllllllll}
\hline \hline Sample & $\mathrm{a}_{0}[\mathrm{~nm}]$ & $\mathrm{R}_{\mathrm{Si}}[\mathrm{nm}]$ & $\mathrm{n}_{3}[\%]$ & $\mathrm{n}_{4}[\%]$ & $\mathrm{n}_{5}[\%]$ & $\mathrm{N}_{\mathrm{Si}}$ & $\mathrm{N}_{\mathrm{C}}$ & $\mathrm{N}_{\mathrm{Si}-\mathrm{Si}}[\%]$ & $\mathrm{N}_{\mathrm{Si}-\mathrm{C}}[\%]$ & $\mathrm{N}_{\mathrm{C}-\mathrm{C}}[\%]$ \\
\hline $\mathrm{D}-64$ & 0.8752 & 0.260 & 0.0 & 96.9 & 3.1 & 4.06 & 4.00 & 15.5 & 69.8 & 14.7 \\
\hline B1-64 & 0.8702 & 0.260 & 1.5 & 93.8 & 4.7 & 4.06 & 4.00 & 15.5 & 70.6 & 13.9 \\
\hline $\mathrm{D}-216$ & 1.3086 & 0.260 & 2.0 & 92.0 & 6.0 & 4.12 & 3.96 & 15.4 & 71.5 & 13.1 \\
\hline B1-216 & 1.3056 & 0.250 & 1.8 & 90.3 & 7.9 & 4.12 & 4.00 & 16.4 & 68.8 & 14.8 \\
\hline D-512 & 1.7364 & 0.250 & 1.4 & 90.4 & 8.2 & 4.14 & 3.99 & 17.5 & 67.7 & 14.8 \\
\hline \hline
\end{tabular}



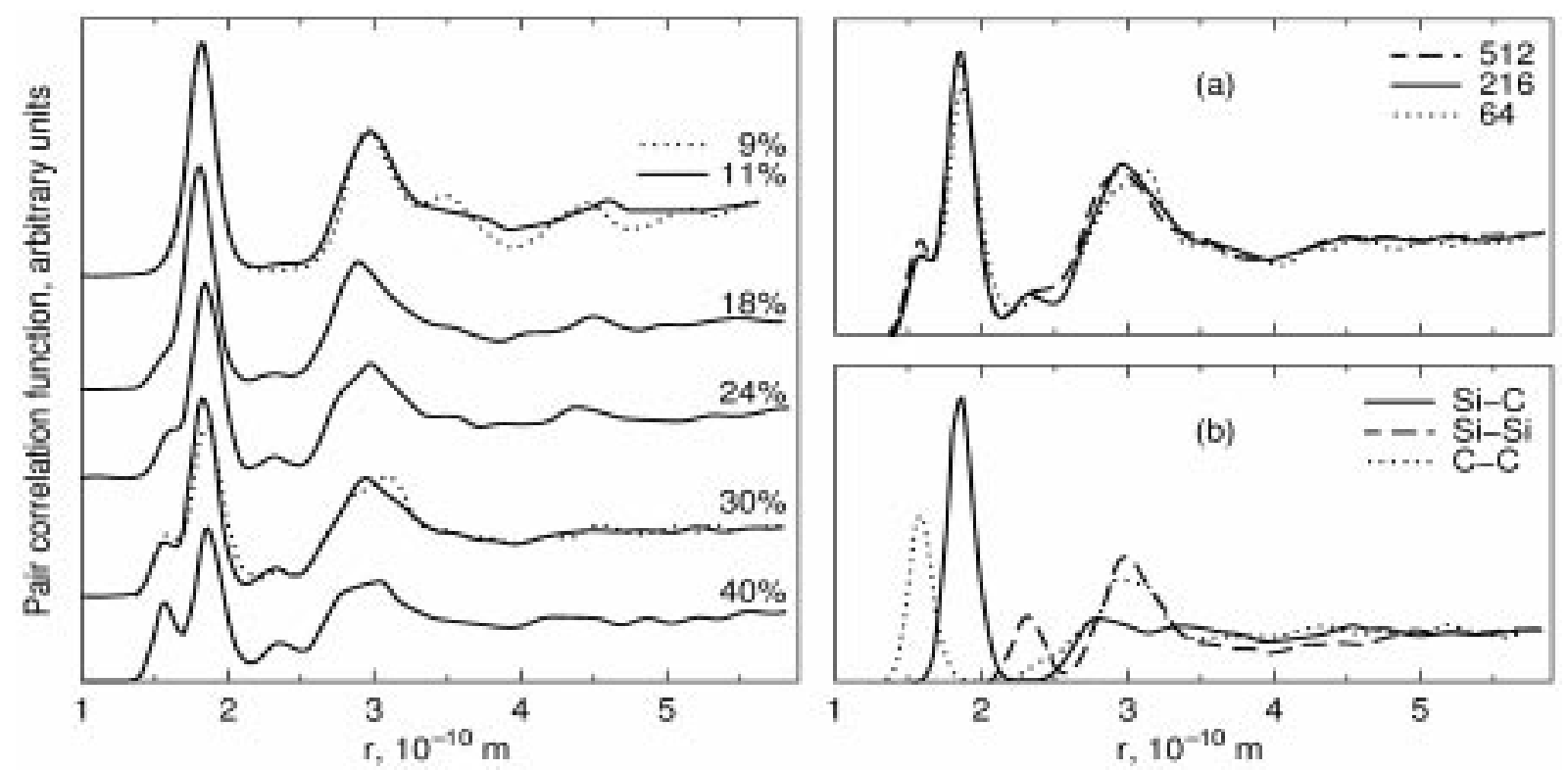

Fig. 1. Calculated pair correlation function (PCF) for a-SiC. The left panel: total PCF of the 64- atom samples (dashed line) and 216atom samples (solid line) with various percentages of homonuclear bonds. The right panel: total PCF for the 64-, 216- and 512-atom samples (a) and partial PCF of the 216-atom sample (b); all the samples have about $30 \%$ of homonuclear bonds.

of the samples with various extents of chemical ordering having approximately $0-2 \%$ and $3-5 \%$ of three-fold $\left(T_{3}\right)$ and five-fold $\left(T_{5}\right)$ coordinated atoms, respectively. Almost all the 64-atom samples have $97 \%$ of four-fold $\left(\mathrm{T}_{4}\right)$ and $3 \%$ of $\mathrm{T}_{5}$ atoms. There is only four-fold coordination in the sample with $8 \%$ of homopolar bonds. From Fig.1 and Table 1, it follows that our results are not very sensitive to the initial structure, quench rate or sample size. Here, we focus our attention on the analysis of the samples with about $30 \%$ of homopolar bonds. The partial C-C PCF ( $\mathrm{g}_{\mathrm{C}-\mathrm{C}}$ ) has an intense first peak at $0.157 \mathrm{~nm}$, to be compared with the nearestneighbor distance in diamond $(0.155 \mathrm{~nm})$. Like to the results of Finocchi et al. [2] and Tersoff [5], we found one secondary maximum at $0.296 \mathrm{~nm}$ corresponding to $\mathrm{C}$-Si-C configuration and a shoulder at $\sim 0.276 \mathrm{~nm}$ associated with C-C-C correlation. Recall that the sample [2] contained about $40-45 \%$ of the carbon homonuclear bonds. In our case, no more than $15 \%$ of $\mathrm{C}$ homonuclear bonds have been found (Table 1). Moreover, unlike [2,4], these bonds are formed by $\mathrm{T}_{4}$ atoms. The partial coordination number of $\mathrm{C}_{\mathrm{C}-\mathrm{C}}$, as computed from the integral of $\mathrm{g}_{\mathrm{C}-\mathrm{C}}$ up to its first minimum, is 1.16. The C-Si PCF has a peak at $0.186 \mathrm{~nm}$ and a second peak at $0.278 \mathrm{~nm}$, with $\mathrm{n}_{\mathrm{Si}-\mathrm{C}}=2.8$. Thus, each $\mathrm{C}$ atom has on average about $\mathrm{n}=3.96$ neighbors (due to several three-fold coordinated atoms), which is close to $\mathrm{n}=4$ in the crystal. The $\mathrm{g}_{\mathrm{Si}-\mathrm{Si}}$ curve has a broad range of neighbor distances. The sharpness of the dip in the Si-Si PCF at around $0.26 \mathrm{~nm}$ (as in Ref. 5 near $0.28 \mathrm{~nm}$ ) is an artifact of the cutoff of the potential $[5,6]$. We have defined that $\mathrm{n}_{\mathrm{Si}-\mathrm{Si}}$ is constant and equal to 1.28 , by varying the cutoff distance $\mathrm{R}_{\mathrm{Si}-\mathrm{Si}}$ in the range of $0.25-0.26 \mathrm{~nm}$. It means that a silicon atom is surrounded on average by 4.12 neighbors, due to 13 stable $\mathrm{T}_{5}$ silicon atoms, which has been found to be present in the D-216 sample. For comparison, the D64 sample is characterized by mean C-C, Si-C and SiSi first-neighbor distances of $0.1594,0.1896,0.2282 \mathrm{~nm}$ and standard deviations of bond lengths of 0.0070 , 0.0079 and $0.0122 \mathrm{~nm}$, correspondingly. The B1-512 sample has 7 three-fold, 463 four-fold and 42 five-fold coordinated atoms. In all the samples no five-fold coordinated carbon atoms have been revealed. Thus, the main feature of our model structures is the prevalence of overcoordination over undercoordination and the presence of stable $\mathrm{T}_{5} \mathrm{Si}$ atoms in all the samples. We have succeeded in obtaining the 64-atom sample with $8 \%$ of homonuclear bonds and without coordination defects.

Fig. 1 illustrates the tendency in the variation of the PCF with varying the extent of chemical ordering in a-SiC. The peaks of C-C and Si-Si correlations at $0.155 \mathrm{~nm}$ and $0.233 \mathrm{~nm}$, respectively, weaken with increasing chemical ordering. In the sample with maximum chemical ordering, Si-C correlations are dominant.

Fig. 2 displays the calculated electronic density of states for the stoichiometric 64-atom and 216-atom samples with various percentages of homonuclear bonds. All the 64-atom samples contain two five-fold coordinated silicon atoms, with the exception of the sample with $8 \%$ of homonuclear bonds, which has no coordination defects. The 216-atom samples have about 3$5 \%$ of three-fold and five-fold coordinated atoms. Other kinds of disorder, such as variations in bond angles and bond lengths, other type of clustering configurations of homopolar bonds, are more or less the same in 

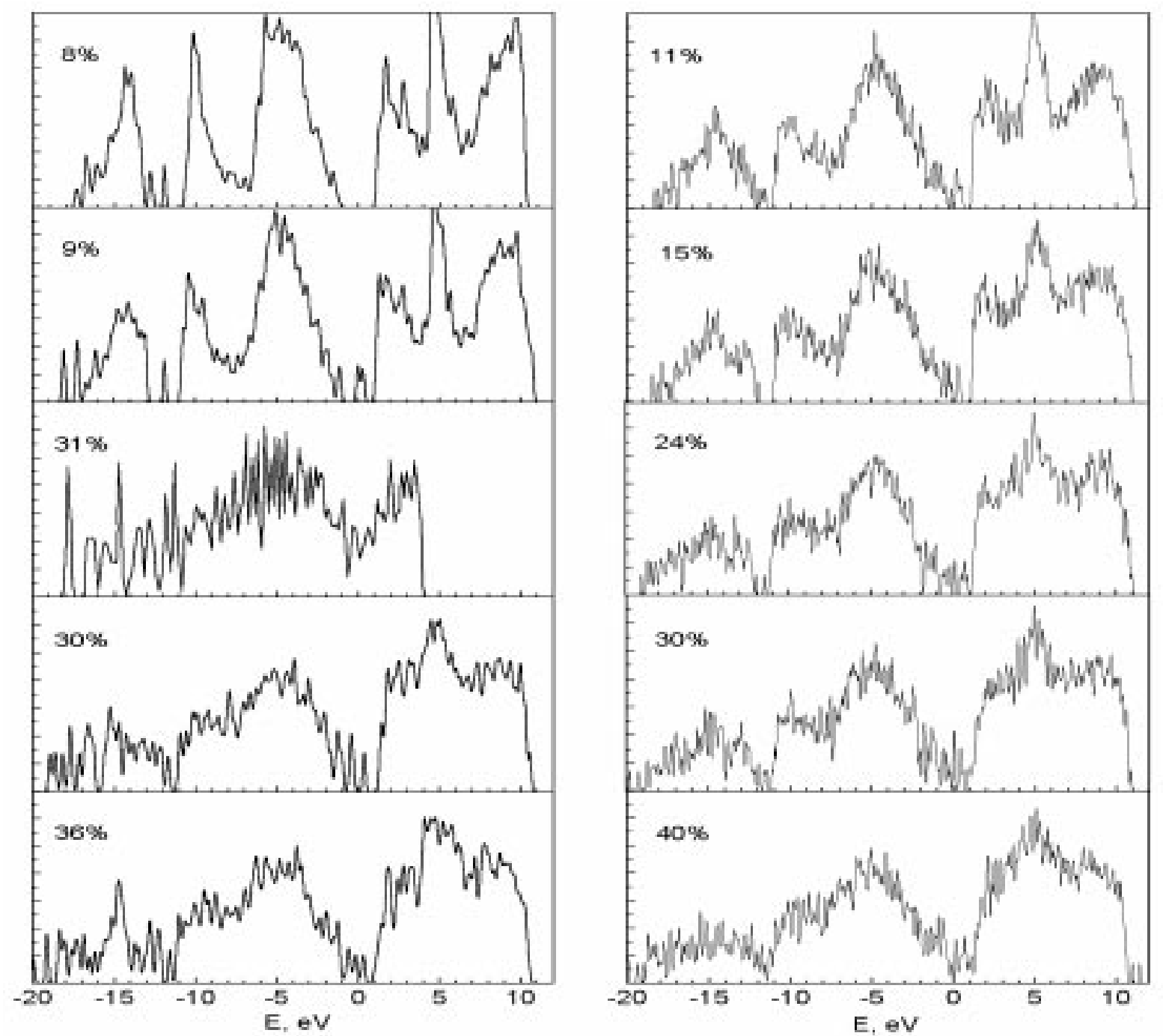

Fig. 2. Total density of states (in arbitrary units) of the 64-atom samples (left panel) and the 216-atom samples (right panel). For comparison, we present the DOS of the 16-atom sample with $31 \%$ of homopolar bonds calculated in the framework of the pseudopotential formalism [9] (left panel).

both sets of the samples. For both sets of the samples the outline of the gap becomes more distinct on going to the alloys with the high extent of chemical ordering. But along with this, the situation takes place when rising ordering does not result in widening the band gap. The latter shows that, besides antisite defects, other structural defects play the very important role in the process of the band gap formation. The effect of introducing homopolar bonds is shown to be a drastic wideness of the s-like low energy valence band. An interesting feature in our DOS is the existence of the ionicity gap at about $-11 \mathrm{eV}$, separating the low-lying valence states from the middle region of the DOS. The tendency in the formation of the ionicity gap with varying the number of homopolar bonds is the same as that of the semiconducting gap. This is consistent with the findings [9], and is in contrast with the results [2], where no the ionicity gap was found.

To explain the mentioned peculiarities of the elec- tronic spectra of our samples, it is reasonable to carry out the analysis of the local density of states. For this purpose, the low defect 64-atom sample with $9 \%$ of homonuclear bonds was singled out. The local DOSs of this sample are shown in Fig. 3.

From Fig. 3 a,b, it is seen that homonuclear silicon bonds give rise to the gap peak localized at $\sim-1.0 \mathrm{eV}$. Gap states related to homonuclear carbon bonds were not revealed (Fig. 3 c,d). To understand the origin of homonuclear bond states in the gap bottom, we have performed the calculation of the local DOSs of isolated $\mathrm{Si}-\mathrm{Si}$ and $\mathrm{C}-\mathrm{C}$ bonds in $3 \mathrm{C}-\mathrm{SiC}$ taking into account with the lattice relaxation. The result of such calculations is presented in Fig. 4. The Si-Si bond gives no gap states but modifies the DOS near the gap. It gives the resonance states at $-1.6 \mathrm{eV}$ and $-12.0 \mathrm{eV}$. Consequently, a reduction of the symmetry caused by defects has to lead to the split of the resonance peak near the gap, which gives rise to gap states at about 
V.I. Ivashchenko et al.: Atomic and electronic structure of a-SiC

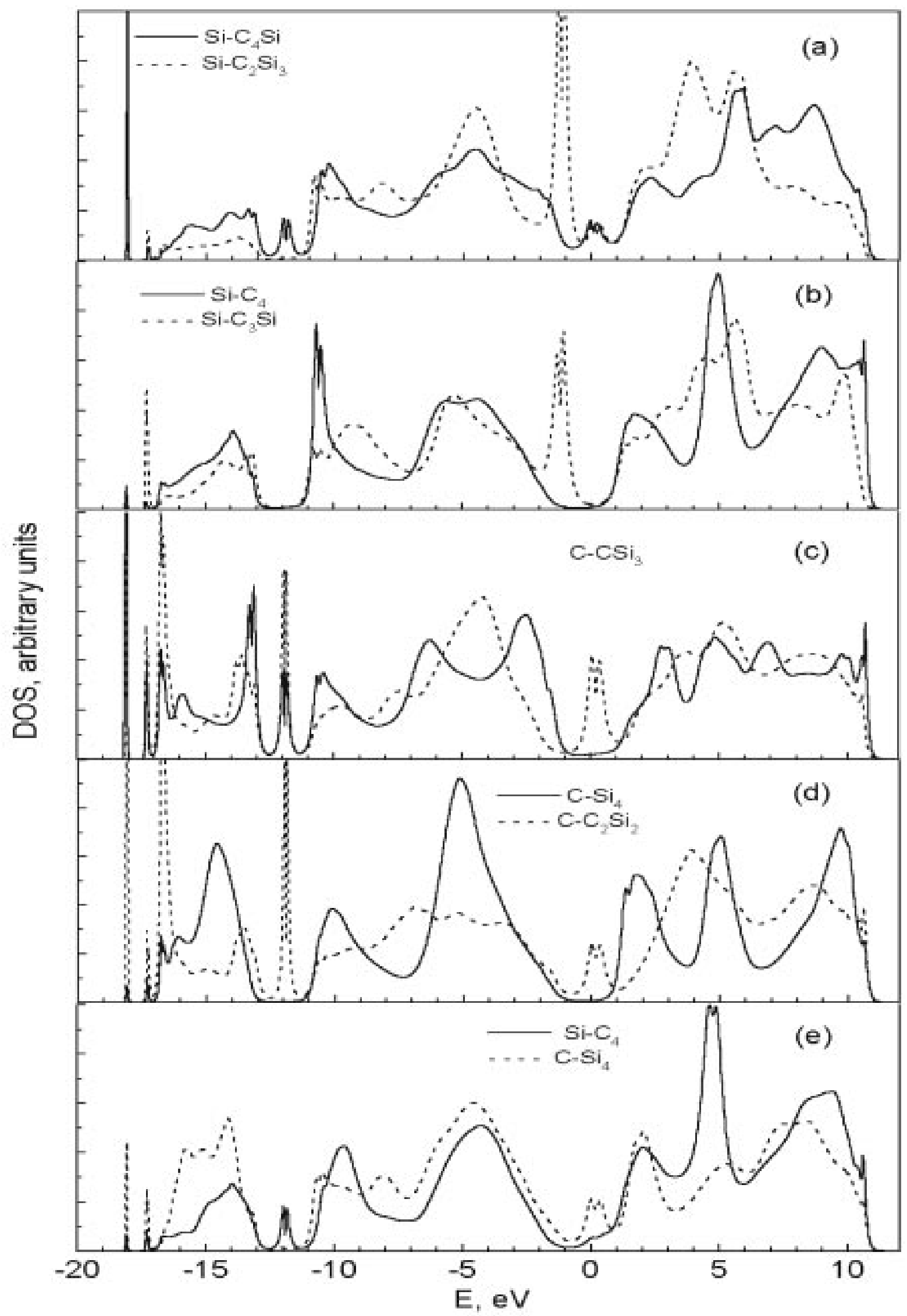

Fig. 3. Local DOSs of the 64-atom sample with $9 \%$ of homonuclear bonds. 
V.I. Ivashchenko et al.: Atomic and electronic structure of a-SiC
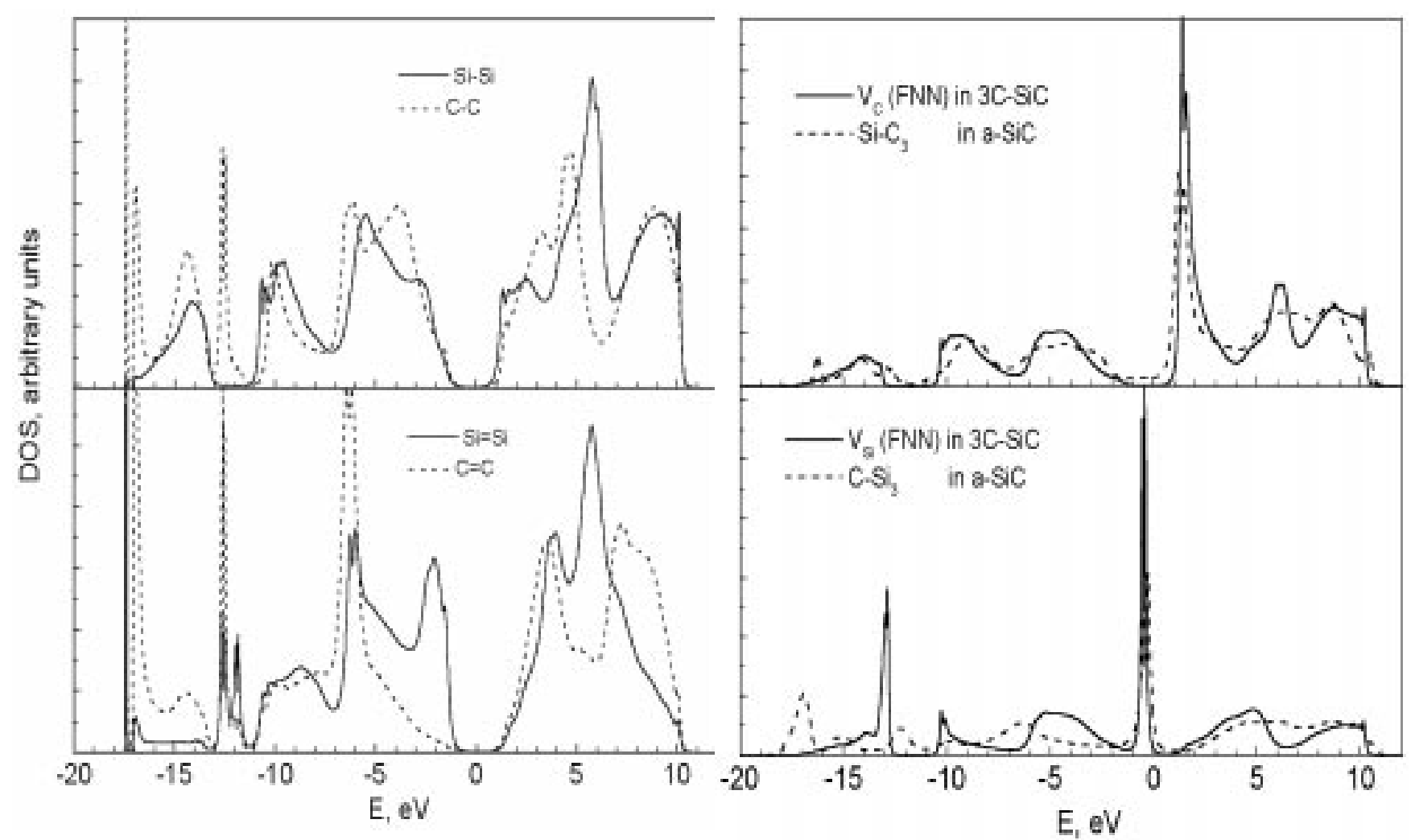

Fig. 4. Calculated local DOSs of $\mathrm{Si}-\mathrm{Si}, \mathrm{C}-\mathrm{C}, \mathrm{Si}=\mathrm{Si}$ and $\mathrm{C}=\mathrm{C}$ bonds (left panel) and the first nearest neighbors $(\mathrm{FNN})$ of a single vacancy in 3C-SiC (right panel). Local DOSs of three-fold coordinated atoms in the 216-atom sample are also shown (right panel).
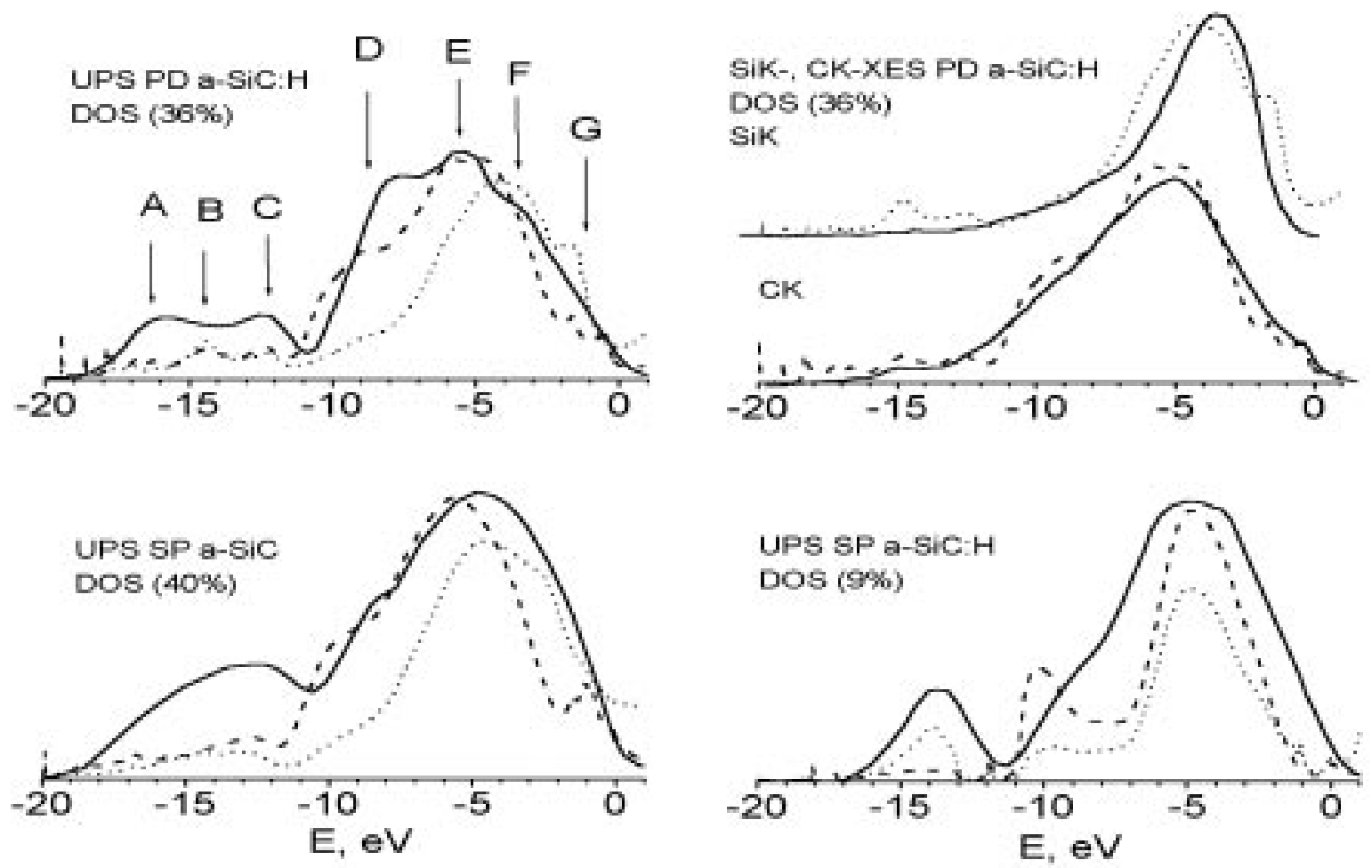

Fig. 5. Superposition of the UPS [20] and XES [21] of plasma-deposition a-SiC:H, the UPS of sputtered a-SiC [22] and sputtered a$\mathrm{SiC}: \mathrm{H}$ [23] (solid line) with the Cp (dashed line) and Sip (dotted line) local DOS of our samples having different percentages of homonuclear bonds. 


\section{V.I. Ivashchenko et al.: Atomic and electronic structure of a-SiC}

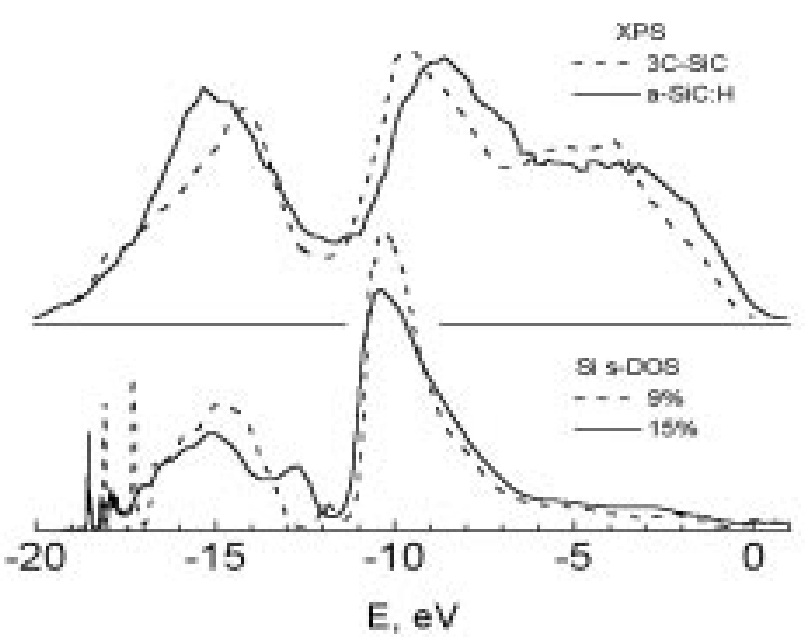

Fig.6. XPS of plasma-deposited a-SiC:H films [24] and siliconlike s-partial DOSs of our samples with different percentage of homonuclear bonds.

$0.2 \mathrm{eV}$ above the valence band top. C-C bonds do not create gap states, but introduce a peak in the C-like band and in the ionicity gap.

Thus, generalizing the results presented in Figs 2, 3 and 4 , one can conclude that the increase of the number of homonuclear bonds in a-SiC lead to the following:

-Widening the C-like band.

-Increase of the number of gap states in the ionicity gap.

-Increasing and broadening the gap peak at $\sim 1.0 \mathrm{eV}$.

-Increase of the number of weak bond atoms, which give rise to gap states.

Homonuclear bonds almost do not modify the gap in a-SiC alloy without coordination defects. Only Si-Si interactions are able to alter the gap width. Consequently, homonuclear Si-Si bonds generate GS provided coordination defects or other kinds of defects are present in the amorphous alloy. Fig. 3 demonstrates that floating and weak bonds connected with $\mathrm{T}_{5}$ and $\mathrm{T}_{4}$ atoms, respectively, cause the appearance of GS at about $0.3 \mathrm{eV}$. We have analyzed the DOS of other 64-atom samples and found that $\mathrm{T}_{5}$ and $\mathrm{T}_{4}$ atoms give also rise to GS at about $-0.4 \mathrm{eV}$. These gap states can be also found in the DOS of the 216-atom samples with the high extent of chemical ordering (Fig. 2).

Since $\mathrm{T}_{3}$ atoms are absent in the small samples, we have calculated the local DOSs of these atoms in the D-216 sample (Table 1). The results, presented in Fig. 4, show that $\mathrm{Si}$ and $\mathrm{C}$ dangling bonds give the strongly localized peaks at $1.2 \mathrm{eV}$ and $-0.2 \mathrm{eV}$, correspondingly. One can see that the dangling bond peaks in a-SiC are located closer to the midgap compared to those in the relaxed $3 \mathrm{C}-\mathrm{SiC}$ crystal.

For a $T_{5}$ atom the obtained results can be interpreted as follows. The four s- and p-orbitals on the $\mathrm{T}_{5}$ atom should be occupied. The extra floating bond representing the fifth linear combination of $\mathrm{sp}^{3}$ hybrids then results in an unpaired electron spin on the neighboring atoms. There are two cases in the distri- bution of electronic states among the five neighbors. In the first case, the unpaired electron is delocalized and can resonate between three or four neighbors, which leads to diffuse states in the gap [14]. In the second case, the "fifth" atom is favored with about $50 \%$ of the wave function, with the rest shared equally by the other four. Such distribution is uneven, but one of the five atoms is usually favored with a large percentage $[15,16]$. As a result, the fifth hybrid remains largely unbonded and has an energy level in the gap, close to the dangling-bond level. It is the latter situation that takes place in our picture of floating bonds, which is close to the conclusions on a-Si $[16,17]$ and is inconsistent with the result of the investigation of coordination defects in a-Si [14].

Strongly disordered $\mathrm{T}_{4}$ atoms (weak bonds) can be considered as $\mathrm{T}_{5}$ atoms, if one adopts that one of the four neighbors with the large bond-angle deviation in the $\mathrm{T}_{4}$ configuration plays the role of the "fifth" atom in the $\mathrm{T}_{5}$ configuration.

Finally, in our samples, we have also revealed GS at the midgap which are formed by the atoms with almost ideal tetrahedral coordination (Fig. 3 e, Si-C 4 ). However, these atoms are the FNN of the abnormally coordinated atoms. Therefore, GS are caused by the creation of the hybrid $T_{4}-T_{3}$, or $T_{4}-T_{5}$ states. These hybrid-bond states, representing weak-bond states, have the same origin as GS originated from the second nearest neighbors of a single vacancy in 3C-SiC [18]. From the energetic localization of floating-, weak- and dangling-bond states (Fig. 3,4), it follows that floating and weak bonds have substantially less localized wave functions than dangling bonds.

Here, we have to stress that our MD simulations are not based on the first-principle formalism, and the DOS calculations are not self-consistent. This is the shortcoming of our investigation. But, the use of the empirical potential in MD simulations allowed us to calculate different sets of the large sized samples with various kinds of defects, which is not possible in a firstprinciple MD simulation. The investigation [19] gives us the basis to believe that the influence of the selfconsistent procedure in determining the DOS of such semiconductor material as a-SiC will be inconsiderable. The latter is also confirmed by the result of the comparison of the DOSs obtained in the framework of the PDF [9] and by using our tight binding approach (Fig. 2). One can see from Fig. 2 that both the spectra agree rather well. But, unlike the pseudopotential method, the tight binding model gives the more distinct gap. This defect of the PDF based methods was discussed by us earlier.

\section{Comparison with experiment}

In order to verify the obtained results on the electronic structure of a-SiC, we have performed the superposition of the ultraviolet and X-ray 


\section{V.I. Ivashchenko et al.: Atomic and electronic structure of a-SiC}

photoemission spectra (UPS, XPS) and X-ray emission spectra (XES) of a-SiC and a-SiC:H films prepared by a plasma deposition, sputtering and reactive sputtering [20-24] with the local partial DOSs of our samples. Photoemission measures the valence band DOS, weighted by a photon energy and atomic-orbital-dependent cross-section. The $\mathrm{C} 2 \mathrm{p}$ states are pronounced in the UPS, while the Si s states dominate in the XPS. The Si and $\mathrm{C}$ p states are reflected in the Si K and C K $\mathrm{XES}$, respectively. The results of the comparison of the theoretical and experimental spectra are presented in Figs 5 and 6 . We have singled out several characteristic peculiarities in the experimental spectra to be interpreted. The peaks $\mathrm{A}$ and $\mathrm{C}$, located at about -17 $\mathrm{eV}-12 \mathrm{eV}$, respectively, are related to $\mathrm{C}-\mathrm{C}$ homonuclear bonds. The peculiarity $\mathrm{B}$ at about 14-15 $\mathrm{eV}$ is mainly originated from the $\mathrm{C} \mathrm{s}$-states that form $\mathrm{Si}-\mathrm{C}$ bonds. Owing to a hybridization effect, there is a small admixture of Si s-states in the spectral range of the peak B (Fig.6). The peak D (at 8-10 eV) has the Si $\mathrm{s}-\mathrm{C} \mathrm{p}$ character. Homonuclear $\mathrm{C} \mathrm{p}-\mathrm{C} \mathrm{p}$ bonds form the structure E at about 5-6 eV (Fig. 4). Using the results presented in [3] and in Fig. 5, we suppose the knee located in the range of $2.5-4 \mathrm{eV}$ to be caused by $\mathrm{Si}$ p-Si $\mathrm{p}$ homonuclear bonds and $\mathrm{Si} \mathrm{p}-\mathrm{C} \mathrm{p}$ heteronuclear bonds. Finally, the knee $\mathrm{G}$ at around $-1.0 \mathrm{eV}$ is mainly formed by homonuclear Si p-Si p bonds (Fig. 3,4). According to the results [3,20-22], hydrogen modifies the experimental spectra in the low energy range inconsiderably. The presence of hydrogen in the films is mostly reflected in strengthening the peaks $\mathrm{D}$ and $\mathrm{E}$ due to forming $\mathrm{C}-\mathrm{H}$ and $\mathrm{Si}-\mathrm{H}$ bonds, respectively. Taking into consideration the latter circumstance, one can deduce that the calculated electronic spectra reproduce somewhat correct the experimental spectra. In superposing the spectra we have used the DOS of the samples containing different amounts of homonuclear bonds. This enabled us to evaluate the degree of chemical ordering of the films. Fig. 5 demonstrates that the increase of an amount of heteronuclear bonds in the theoretical samples results in broadening the ionicity gap, narrowing the carbon-like s-band and decreasing the distance between the Si-p and C-p peaks (the E-F distance). From

\section{References}

1. J. Bullot and M.P. Schmidt, Phys. Status. Solidi (b) 143 ( 1987) $345-418$

2. F. Finocchi, G. Galli and M. Parinello, C.M. Bertoni, Phys. Rev. B 68 (1992)

$$
\text { 3044-3047. }
$$

3. J. Robertson, Phil. Mag. B 66 (1992) 615-638.

4. P.C. Kelires, Phys. Rev. B 46 (1992) 10048-10061.

5. J. Tersoff, Phys. Rev. B 49 (1994) 16349-16352.

6. J. Tersoff, Phys. Rev. B 39 (1989) 5566-5568.

7. P.C. Kelires and P.J.H. Denteneer, J. Non-Cryst. Solids 231 (1998) 200-204.

8. H. Efstathiadis and Z. Yin, F.W. Smith, Phys. Rev. B 46 (1992) 13119-13130. this we deduce that the distinct dip at $\sim-11.0 \mathrm{eV}$ and the small width of the peaks at $\sim-5.0 \mathrm{eV}$ in the experimental spectra are characteristic features of the films with the high extent of chemical ordering. Such features are distinctly expressed in the spectrum of the sp a-SiC:H film, consequently, it has higher chemical ordering than other films.

\section{Conclusion}

We have studied in this paper the short-range problem in stoichiometric silicon carbide by calculating the atomic and electronic structures among various configurations of order. The results reveal that, in a$\mathrm{SiC}$, the Si-Si homonuclear bonds give rise to defect states at the ionicity gap, separating the low-lying valence states from the middle region of the spectrum, and the bottom of the semiconducting gap, which causes the decrease of the latter gap. The Si-Si homonuclear bond can give rise to GS, if it is sited in the strongly distorted surrounding. Two peaks of the weak- and floating- bond states are localized in the middle of the gap. Our picture of the floating bond consists of a single dominant state involving an unpaired electron spin with one secondary atom. We have found two strongly localized gap states at the gap bottom and the gap top originated from $\mathrm{C}$ - and Si- dangling bond atoms, respectively. Floating- and weakbond states are substantially less localized than dangling-bond states. The obtained results show that our combination of molecular dynamic empirical simulation and recursion calculations is able to rather correctly describe the atomic and electronic distributions in a-SiC with different extents of short-range order. The suggested band model of a-SiC is in general features confirmed by the results of measurements of ultraviolet photoemission and X-ray emission spectra and other experimental data on amorphous silicon carbide films.

This work was supported partly by the STCU Contract No. 1590.
9. P.C. Kelires and P.J.H. Denteneer, Solid State Commun. 87 (1993) 851-855.

10. R. Haydock, V. Heine and M.J. Kelly, J. Phys. C: Solid State Phys. 5 (1972) 2845-2858.

11. I. Stich, R. Car and M. Parinello, Phys. Rev. B 44 (1991) 11092-11103.

12. P. Vogl, H.J. Hjalmarson and J.D. Dow, J. Phys. Chem. Solids 44 (1983) 365-378.

13. C.M.M. Nex, Comput. Phys. Commun. 43 (1984) 101-119.

14. R. Biswas, C.Z. Wang, C.T. Chan, K.M. Ho and C.M. Soukoulis,

Phys. Rev. Lett. 14 (1989) 1491-1494.

15. S.T. Pantelides, Phys. Rev. Lett. 23 (1986) 2979-2982.

16. J.H. Stathis, Phys. Rev. B 40 (1989) 1232-1242.

17. M. Fornari, M. Peressi, S. de Gironcoli and A. Baldereschi, Europhys. Lett. 47 (1999) 481-486. 


\section{V.I. Ivashchenko et al.: Atomic and electronic structure of a-SiC}

18. V.I. Ivashchenko, V.I. Shevchenko, L.A. Ivashchenko and G.V. Rusakov, J. Phys. C: Condens. Mater. 11 (1999) $3265-$ 3272 .

19. D.A. Papaconstantopoulos and E.N. Economou, Phys. Rev. B 22 (1980) 2903-2907.

20. Y. Katayama, T. Shimada, K.L. Kobayashi, C. Jiang, H. Daimon and Y. Murata J. Non-Cryst. Solids 59 (1983) 561-565.
21. G. Wiech, H. Langer, U. Lepa and A. Simunek, J. NonCryst. Solids 164-166(1993) 1023-1026.

22 J. Tejeda, N.J. Shevchik and M. Cordona, Amorphous and Liquid Semiconductors, ed. By J. Stuke (London: Taylor \& Francis) (1974) 557-562.

23. R.C. Fang, L. Ley, Phys. Rev. B 40 (1989) 3818-3826.

24. T.M. Parril and V.M. Bermudez, Solid State Commun. 63 (1987) 231-235. 